\title{
Growing Resistance to Vancomycin among Methicillin Resistant Staphylococcus Aureus Isolates from Different Clinical Samples
}

\author{
Prakash Chandra Pahadi, ${ }^{1}$ Upendra Thapa Shrestha, ${ }^{1}$ Nabaraj Adhikari, ${ }^{1}$ Pradeep Kumar Shah, ${ }^{2}$ Ritu Amatya ${ }^{3}$ \\ 'Department of Microbiology, Kantipur College of Medical Science, Kathmandu, Nepal, ${ }^{2}$ Department of Microbiology, Tri- \\ Chandra Multiple Campus, Kathmandu, Nepal, ${ }^{3}$ Department of Microbiology, Nepal Medical College, Kathmandu, Nepal.
}

\section{ABSTRACT}

Introduction: Methicillin resistant Staphylococcus aureus (MRSA), majorly associated with nosocomial and community infections worldwide, are emerging as resistant strains to many antibiotics narrowing down the efficacy of antimicrobial therapy. In order to investigate the changing resistant pattern of MRSA to empirical drugs, the study was carried out at KIST Medical College and Hospital, Nepal. It also aims to determine the minimum inhibitory concentration (MIC) of vancomycin among MRSA.

Methods: Altogether 3500 clinical samples including 1303 blood, 1489 urine and 708 body fluids were collected and processed. Isolated S. aureus were further screened for methicillin resistance by Kirby-Bauer disk diffusion technique using cefoxitin (30 $\mu \mathrm{g})$ disk. All MRSA were subjected to in vitro determination of MIC of vancomycin by agar dilution method as recommended by CLSI guidelines.

Results: Total 287 S. aureus were isolated from the different clinical samples. Altogether 248 $(86.41 \%)$ were found to be multidrug resistance (MDR) while $42(14.63 \%)$ of the isolates were methicillin resistance with the highest prevalence in the age group of 16-30. All $42(100 \%)$ MRSA isolates were resistant to ampicillin and penicillin followed by 41 (97.62\%), 32 (76.19\%), $31(73.81 \%), 29(69.05 \%), 9(21.43 \%)$ and seven $(16.67 \%)$ to cefotaxime, gentamycin, cotrimoxazole, erythromycin, tetracycline and ciprofloxacin respectively. Although all MRSA strains were sensitive to vancomycin on disc diffusion, four isolates were intermediates in vitro determination of MIC of vancomycin. The break point for vancomycin was found to be $15 \mathrm{~mm}$.

Conclusions: The increment in vancomycin MIC among MRSA is alarming. Strict control measures to prevent MRSA spread and a routine surveillance for VRSA must be incorporated in hospitals.

Keywords: $m d r ; m r s a ; m i c ;$ visa; vrsa.

\section{INTRODUCTION}

The first methicillin resistant $S$. aureus, MRSA was reported in 1961 in UK. ${ }^{1}$ Until the explosion of MRSA threat in hospital in 1990s, it generally remained an uncommon finding even in hospital settings. ${ }^{2}$ Soon, several outbreaks of MRSA infections occurred worldwide..$^{3,4}$ The global estimate showed that around two billion people carry some forms of S. aureus; of

Correspondence: Mr. Prakash Chandra Pahadi, Department of Microbiology, Kantipur College of Medical Science, Sitapaila, Kathmandu, Nepal. Email: pahadiprakash@gmail.com, Phone: +977. 9841852678 
these 53 million $(2.7 \%$ of carriers) are thought to carry MRSA. ${ }^{5}$

MRSA has remained sensitive to only empirical drug vancomycin, the glycopeptides for few years ${ }^{3}$ but soon in 1997, the first isolates of $S$. aureus with reduced susceptibility to vancomycin (MIC, $8 \mu \mathrm{g} / \mathrm{ml}$ ) were reported in Japan. ${ }^{6}$ Since then, six confirmed vancomycin - resistant S. aureus (VRSA) (MIC, $\geq 32 \mu \mathrm{g} /$ $\mathrm{ml}$ ) have been documented in United States. ${ }^{7}$

Although no VRSA is reported in Nepal till now, the emergence of MRSA isolates with reduced susceptibility to vancomycin reinforced the importance of growing research in this area. Thus the study was carried with an objective to know the changing resistant pattern of MRSA isolates to vancomycin.

\section{METHODS}

The study was descriptive cross-sectional study which was carried out in KIST Medical College and Hospital, Imadol, Lalitpur from November 2011 to May 2012.

The research was approved by Institutional Research Committee, IRC board of the hospital. Both the verbal and written consent were obtained from the patient and their care takers prior to sample collection as stated by IRC.

All patients suspecting of MRSA infections and recommended by physician for their sample culture were included in the study. Those patients who refused to participate in the study and didn't provide verbal and written consent were excluded from the study.

Altogether 3500 samples were collected, out of which 1303, 1489 and 708 were blood, urine and body fluids respectively. The collected samples were processed immediately, within an hour. All the blood samples were cultured in Brain heart infusion (BHI) broth while other samples in blood agar (BA) plates and incubated aerobically at 37ロC for 24-72 hours. 0-haemolytic or typical colonies on blood agar and cultures from BHI were further screened for identification of $\mathrm{S}$. aureus following standard conventional procedures. S. aureus ATCC 25923 was used as quality control reference strain.

Methicillin resistance was tested with Kirby-Bauer disk diffusion technique using cefoxitin $(30 \mu \mathrm{g})$ disk. The diameter for zone of inhibition on Muller Hilton agar was measured after incubation at $35^{\circ} \mathrm{C}$ for 24 hours. Strains with zone of diameter $\leq 21 \mathrm{~mm}$ were regarded as methicillin resistant . ${ }^{6}$ All the identified isolates of $\mathrm{S}$. aureus were subjected to in-vitro antibiotic susceptibility testing by Kirby-Bauer disk diffusion method. The antibiotics used were penicillin (10 units), gentamycin
(10 $\mu \mathrm{g})$, ampicillin $(10 \mu \mathrm{g})$, ciprofloxacin $(5 \mu \mathrm{g})$, erythromycin $15 \mu \mathrm{g})$, tetracycline $(30 \mu \mathrm{g})$, cotrimoxazole $(25 \mu \mathrm{g})$, cefotaxime $(30 \mu \mathrm{g})$, vancomycin $(30 \mu \mathrm{g})$.

All the identified MRSA isolates thus obtained were subjected to in vitro determination of minimum inhibitory concentration (MIC) of vancomycin by agar dilution method as recommended by CLSI guidelines. ${ }^{8}$

Raw data obtained from laboratory investigation were tabulated and presented in defined tables and in graphs to explore the findings. WHO NET version 5.1 tool was used for data analysis.

\section{RESULTS}

Out of 3500 clinical samples from inpatients and outpatients visiting KIST Hospital, $287 \mathrm{~S}$. aureus were isolated. Among 287 total S. aureus, the highest number of S. aureus were isolated from urine sample $109(38 \%)$ followed by $92(32 \%)$ and 86 (30\%) from blood and body fluid respectively. The prevalence of $\mathrm{S}$. aureus was found to be slightly higher in hospitalized patients which were $151(52.61 \%)$ than that of outpatients 136 (47.39\%). However both the sexes were equally infected.

A highest percentage of S. aureus isolates 274 (95.47\%) were resistant to penicillin followed by $273(95.12 \%)$ to ampicillin. Similarly 134 (46.69\%), 122 (42.51\%), 110 (38.32\%), 110 (38.32\%), 108 (37.63\%), 84 (29.27\%), and $42(14.63 \%)$ of $S$. aureus were resistance to erythromycin, gentamycin, cotrimoxazole, tetracycline, ciprofloxacin, cefotaxime and cefoxitin respectively. All the isolates were sensitive to vancomycin. About 248 (86.41\%) of those isolates were MDR and 42 (14.63\%) were MRSA. All MRSA were multidrug resistant. The higher percentage of MRSA was found in body fluid samples (Figure 1).

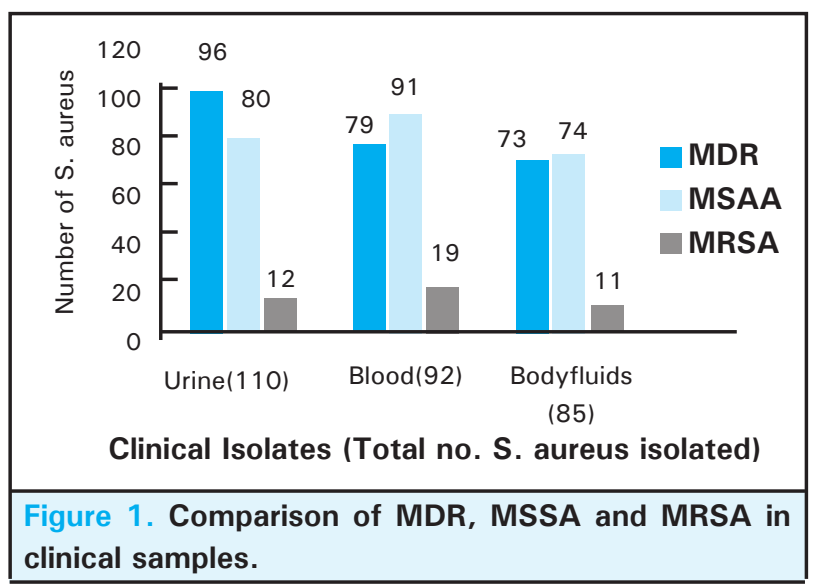


Pahadi et al. Growing Resistance to Vancomycin among Methicillin Resistant Staphylococcus Aureus Isolates from Different Clinical Samples

Regarding methicillin sensitive S. aureus (MSSA), $245(100 \%)$ were sensitive to vancomycin followed by cefotaxime 201 (82.04\%), and gentamicin 165 $(67.35 \%)$. Similarly, 42 (100\%) MRSA were sensitive to vancomycin followed by $35(83.33 \%)$ to ciprofloxacin (Table 1).

\begin{tabular}{|c|c|c|c|c|}
\hline \multirow[t]{2}{*}{ Antibiotics used } & \multicolumn{2}{|c|}{ Number of sensitive (\%) } & \multicolumn{2}{|c|}{ No. of MDR among } \\
\hline & MRSA to & MSSA to & \multirow[t]{6}{*}{ MRSA (\%) } & \multirow[t]{6}{*}{ MSSA (\%) } \\
\hline Ampicillin $(10 \mu \mathrm{g})$ & $0(0)$ & $13(5.31)$ & & \\
\hline Cefotaxime $(30 \mu \mathrm{g})$ & $1(2.38)$ & 201 (82.04) & & \\
\hline Cotrimoxazole $(25 \mu \mathrm{g})$ & $31(73.81)$ & $135(55.10)$ & & \\
\hline Ciprofloxacin $(5 \mu \mathrm{g})$ & 35 (83.33) & $143(58.37)$ & & \\
\hline Erythromycin $(15 \mu \mathrm{g})$ & $13(30.95)$ & $140(57.14)$ & & \\
\hline Gentamycin $(10 \mu \mathrm{g})$ & $10(23.81)$ & $165(67.35)$ & \multirow[t]{4}{*}{$42(100)$} & \multirow[t]{4}{*}{206 (84.08) } \\
\hline Penicillin (10 units) & $0(0)$ & $14(5.71)$ & & \\
\hline Tetracycline $(30 \mu \mathrm{g})$ & $33(78.57)$ & $142(57.96)$ & & \\
\hline Vancomycin $(30 \mu \mathrm{g})$ & $42(100)$ & $245(100)$ & & \\
\hline Total number & 42 & 245 & 42 & 245 \\
\hline
\end{tabular}

Out of 42 MRSA isolates, 4 (9.52\%) isolates were intermediate to vancomycin growing in $4 \mu \mathrm{g} / \mathrm{ml}, 5$ $(11.90 \%)$ isolates grew in $2 \mu \mathrm{g} / \mathrm{ml}, 5(11.90 \%)$ isolates grew in $1 \mu \mathrm{g} / \mathrm{ml}$ and 28 (66.67\%) grew in only $0.5 \mu \mathrm{g} /$ $\mathrm{ml}$ as shown in the scatter-plot below. The breakpoint for vancomycin was found to be $15 \mathrm{~mm}$ (Figure 2).

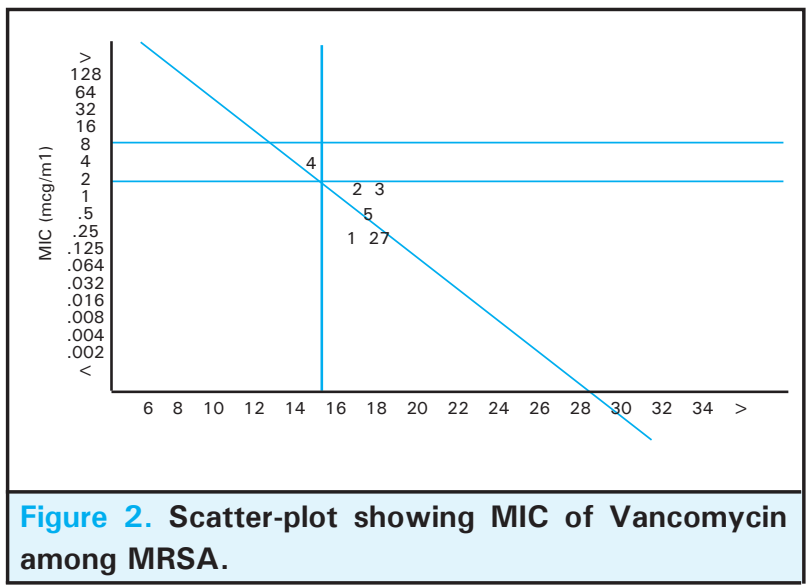

\section{DISCUSSION}

Staphylococcus aureus is one of the key microorganisms involved in nosocomial infections as well as in the community settings. ${ }^{9}$ The emerging resistant strains of $\mathrm{S}$. aureus and its occurrence as an infectious agent in many clinical infections have been a cause of great threat to the world. It also reinforced the importance of detail studies on such areas ${ }^{10,11}$ and our study showed the prevalence of $\mathrm{S}$. aureus in different clinical samples was $8.20 \%$ with a slightly higher percent in urine samples. The study by Daniyan and Sani reported even less percent of $\mathrm{S}$. aureus which accounts $4.52 \% .^{12}$ The prevalence was reported higher such as $30 \%$ and
$36.8 \%$ respectively in the study by Taiwo and Gerald et al. in Cameroon. ${ }^{13,14}$ It shows that the prevalance of $\mathrm{S}$. aureus may vary from one hospital to another.

All the isolates of $\mathrm{S}$. aureus were categorized into two groups: methicillin resistant $S$. aureus (MRSA) and methicillin sensitive S. aureus (MSSA). Out of $287 \mathrm{~S}$. aureus, $86.41 \%$ were multidrug resistant and $14.63 \%$ were MRSA. All MRSA were the multi drug resistant. The study done by Rajbhandari et al. in Bir Hospital reported $55 \%$ MRSA infection in outpatients and $76 \%$ in hospitalized patients. ${ }^{15}$ While Adebayo and Johnson, and Dar et al. reported $26.9 \%$ and $54.85 \%$ prevalence of MRSA respectively in their studies. ${ }^{16,17}$ Khanal and Jha found $68 \%$ MRSA from skin infected patients attending the hospitals in Chitwan Nepal. ${ }^{18}$ The study by Niraula reported $13.7 \%$ and $12.9 \%$ of MRSA in outpatients and inpatients respectively visiting Manmohan Memorial Community Hospital, Kathmandu. ${ }^{19}$ MRSA infections were found to be varied in infections of different parts of body, geographical regions as well as status of patients. Likewise, Salab et al. reported $63 \%$ of MRSA isolates were multi drug resistant ${ }^{20}$ which is less than in our study $(100 \%$ resistant) indicating the changing of MRSA to multi drug resistant MRSA.

The most sensitive drugs for MSSA were found to be vancomycin $(100 \%)$ followed by cefotaxime $(82.13 \%)$ and gentamicin $(67.48 \%)$ while the most sensitive antibiotics for MRSA were also vancomycin (100\%) followed by ciprofloxacin $(83.40 \%)$ and tetracycline $(78.60 \%)$ in our study. Adebayo and Johnson also reported vancomycin as a choice of drug for MRSA infections. ${ }^{16}$ Shailesh et al. in Pondicherry India reported that MRSA were $100 \%$ sensitive to vancomycin. ${ }^{21}$ The 
results from previous researches indicated the emerging resistant of MRSA towards the vancomycin. The researchers also showed that the penicillin and other 口 -lactam drugs as the least effective drugs in MRSA infections because of the production of $\square$-lactamase enzymes by the pathogens.

Although S. aureus show a zone of inhibition in the sensetive range for vancomycin, MIC determination showed a higher MIC for vancomycin which gives a true picture of the emerging resistance of $\mathrm{S}$. aureus towards this drug.In this study $9.52 \%$ isolates of MRSA were found to be vancomycin intermediates $(4 \mu \mathrm{g} / \mathrm{ml})$ while remaining all are vancomycin sensitive. The prevalence of vancomycin intermediate $S$. aureus (VISA) strains in India was reported to be $6.3 \% .^{22}$ Likewise Tiwari and Sen had reported two strains of VRSA and six strains of intermediate (VISA) in the northern part of India. ${ }^{23}$ The MIC values indicated that only 1.9 per cent isolates were resistant to vancomycin on the study conducted by Venubabu et al. in Hyderabad, India. ${ }^{24}$ Although the prevalence of MRSA in our study was less, the vancomycin intermediates were found to be significantly increasing with a break point of $15 \mathrm{~mm}$. The results showed the higher risk of VRSA in Nepal too.

\section{CONCLUSIONS}

Widespread use of vancomycin to treat infections caused by MRSA and other gram-positive cocci such as Enterococcus spp., S. aureus and Coagulase negative S. aureus (CoNS) had led to the emergence of VISA and VRSA. The large scale of development and subsequent spread of resistance to vancomycin had been perceived as a fearsome threat to the already challenging therapy of MRSA. Hence it is suggested to perform routine MIC of vancomycin in MRSA infections.

\section{ACKNOWLEDGMENTS}

We express our sincere gratitude to all the staffs and faculties of Kantipur College of Medical Science and KIST Medical College and Hospital.

\section{REFERENCES}

1. Jevons MP. Cebenin resistant Staphylococci. Letter BMJ. 1961;1:124-5.

2. Johnson AP, Aucken HM, Cavendish S, Ganner M, Wale MC and Warner M. Dominance of EMRSA-15 and-16 among MRSA causing nosocomial bacteraemia in the UK: analysis of isolates from the European Antimicrobial Resistance Surveillance System (EARSS). J Antimicrob Chemother. 2004;48:143-4.

3. Voss A, Milutovic D, Wallrauch-Schwartz C, Rosdahl VT and Braveny I. Methicillin resistant Staphylococcus aureus in Europe. Eur. J. Clin. Microbiol. Infect. Dis. 1994;13:50-55.

4. Voss A and Doebbling BN. The worldwide prevalence of methicillin-resistant Staphylococcus aureus. Int. J. Antimicrob. Agents. 1995;5:101-6.

5. Graham PL, Lin SX and Larson EL. A US population based survey of Staphylococcus aureus colonization. Ann Intern Med. 2006;144:318 - 25.

6. Hiramatsu K, Aritaka N, Hanaki H, Kawasaki S, Hosoda Y, Hori S, Fukuchi Y and Kobayashi I. Dissemination in Japanese hospitals of strains of Staphylococcus aureus heterogeneously resistant to vancomycin. Lancet. 1997;350:1670-3.

7. Centers for Disease Control and Prevention. Vancomycin resistant S. aureus - Pennsylvania, 2002. Morb Mortal Wkly Rep. 2002;51:902.

8. Clinical Laboratory Standards Institute. Performance standards for antimicrobial susceptibility testing. Twentieth informational supplement ed. CLSI document M100-S20. Wayne, PA: CLSI. 2010.
9. Estivariz CF, Park SY, Hageman JC, Dvorin J, Melish MM, Arpon R, Coon P, Slavish S, Kim M, McDougal LK, Jensen B, McAllister S, Lonsway D, Killgore G, Effler PE and Jernigan DB. Emergence of community-associated methicillin resistant Staphylococcus aureus in Hawaii, 2001-2003. J Infect. 2003;54:349-57.

10. Walsh C. Deconstructing vancomycin. Science. 1999;284:442-3.

11. Appellbaum PC and Bozdogan B. Vancomycin resistance in Staphylococcus aureus. Clin Lab Med. 2004;24:381-402.

12. Daniyan SY and Sani AM. Antibiotics susceptibility of Staphylococcus aureus isolated from some clinical samples in a secondary health care institute, Nigeria. 2001.

13. Taiwo S. Methicillin-resistant Staphylococcus aureus (MRSA) isotypes in Illorin, Nigeria. Afri. J. Clin. Exp. Microbiol. 2004;5(2):189-197.

14. Gerald N, Jane- Francis TK, Akoachere, Lucien HK, Emmanuel D, Nfoncham and Roland N. Staphylococcus aureus isolates from clinical and environmental samples in a semi- rural area of Cameroon: phenotypic characterization of isolates. 2009.

15. Rajbhandari R, Manandhar SP and Shrestha J. Comaparative study of MRSA and its antibiotic susceptibility pattern in indoor and outdoor patients in Bir Hospital, Nepal. Nepalese J Microbiol. 2003;1:62-5.

16. Adebayo SO and Johnson L. Antimicrobial susceptibility patterns and characterization of clinical isolates of Staphylococcus aureus in Kwazulu-Natal province, South Africa. BMC. 2006;6:125. 
17. Dar JA, Thoker MA and Khan JA. Molecular epidemiology of clinical and carrier strains of methicillin resistant Staphylococcus aureus (MRSA) in the hospital settings of North India. Annals of clin Microb and antimicrob. 2006;5:22.

18. Khanal LK and Jha BK. Prevalence of Methicillin resistant Staphylococcus aureus (MRSA) among skin infection cases at a hospital in Chitwan, Nepal. Nepal Med Coll J. 2010;12(4):224-8.

19. Niraula PM. Prevalence of Staphylococcus aureus and Methicillin resistant S. aureus (MRSA) as nasal carrier among outpatients, inpatients and hospital staffs at Manmohan Memorial Community Hospital, Nepal. A Dissertation Submitted to Department of Microbiology, Kantipur College of Medical Science (affiliated to Tribhuvan University), Sitapaila, Kathmandu as a partial fulfillment of the requirements for the award of the degree of Master of Science in Medical Microbiology. 2011.

20. Salab IK, Nagla AA and Ahmed IF. Prevalence and antimicrobial susceptibility pattern of methicillin resistant Staphylococcus in a Sudanese surgical ward.Pharmacology and Pharmacy. ZJMS. 2012;3:103-8.
21. Shailesh K, Noyal MJ, Mariya E, Reecha S, Sivaraman U, Pramodhini S and Srirangaraj S. Prevalence and current antibiogram of Staphylococcus isolated from various clinical specimens in a tertiary care hospital in Pondicherry. The international journal of Microbiology DOI. 2012;10:5580/2A80.

22. Song JH, Hiramatsu $\mathrm{K}$ and Suh JY. The Asian network for Survillance of resistant pathogens (ANSORP) study group. Antimicrob Agents Chemothe. 2004;48(12):4926-8.

23. Tiwari HK and Sen MR. Emergence of Vancomycin resistant Staphylococcus aureus (VRSA) from a tertiary care hospital in northern parts of India. BMC Infect Dis. 2006;6:156.

24. Venubabu T, Channappa TS and Subhaschandra M. Vancomycin resistance among methicillin resistant Staphylococcus aureusisolates from intensive care units of tertiary care hospitals in Hyderabad. Indian J. Med. Res. 2011;134:704-8. 